8 Bjoraker JA, Ryu JH, Edwin MK, et al. Prognostic significance of histopathologic subsets in idiopathic pulmonary fibrosis. Am J Respir Crit Care Med 1998; 157: 199-203.

9 Veeraraghavan S, Latsi PI, Wells AU, et al. BAL findings in idiopathic nonspecific interstitial pneumonia and usual interstitial pneumonia. Eur Respir J 2003; 22: 239-244.
10 MacDonald SL, Rubens MB, Hansell DM, et al. Nonspecific interstitial pneumonia and usual interstitial pneumonia: comparative appearances at and diagnostic accuracy of thin-section CT. Radiology 2001; 221: 600-605.

\title{
Aqua jogging-induced pulmonary oedema
}

\section{To the Editors:}

The case study by WENGER and RUSSI [1], of pulmonary oedema occuring during aqua jogging, is interesting. Aqua jogging certainly lowers the burden to joints and tendons as compared with land running. Conversely, running or cycling at sustained intensity may lead to higher burden to lung tissue in water than on ground. In water, the exercising mechanical strain [2] is strengthened by congestion of pulmonary vessels (hence, decreased lung compliance) and also by the inspiratory loading due to hydrostatic pressure, which is likely to enlarge intraairway pressure swings [3, 4]. In addition, the work of breathing increases progressively during endurance exercise at constant work [5]. Therefore, it seems difficult to believe that stress failure of alveolar or bronchial capillaries is improbable in the case reported by WENGER and RUSSI [1]. In an experiment designed to compare immersed versus ground 30-min cycling, thoracic electrical impedance was lower during recovery on land after exercising in water than on ground, which reflected a larger amount of thoracic fluid, while stroke volume was simultaneously lower, i.e. some degree of suboedema may have been present $[4,6]$. In addition, $20^{\circ} \mathrm{C}$ water carries a cold stress, even to an exercising subject $[4,7,8]$, and even mild cooling increases peripheral vascular resistance, left ventricular afterload and pulmonary congestion [7]. Finally, symptoms related to pulmonary oedema occur earlier during sustained exercising in water than on land. In the case reported by WENGER and RUSSI [1] symptoms occured after $20 \mathrm{~min}$, which matches other reports (see references quoted by WENGER and RUSSI [1], and also recently gathered data [9]). All in all, the occurrence of pulmonary oedema during various conditions of immersed exercising is not rare, which encourages efforts for a better understanding of the underlying pathophysiological mechanisms. Cardiovascular strains linked to sustained exercise during immersion should not be overlooked. Detailed recording of each case's circumstances of occurrence should aid recognition of recurrent features and tracking possible underlying pathways [9].

\section{J. Regnard}

Functional testing - Clinical Physiology, University Hospitals, Besançon, France.

\section{STATEMENT OF INTEREST}

None declared.

\section{REFERENCES}

1 Wenger M, Russi EW. Aqua jogging-induced pulmonary oedema. Eur Respir J 2007; 30: 1231-1232.

2 Dempsey J. Respiratory systems limitation to endurance exercise performance in health. www.ersnet.org/ers/lr/ browse/default.aspx?id=32967 Last updated: September, 2007.

3 Agostoni E, Gurtner G, Torri G, Rahn H. Respiratory mechanics during submersion and negative-pressure breathing. J Appl Physiol 1966; 21: 251-258.

4 Bréchat $\mathrm{PH}$, Wolf JP, Simon-Rigaud ML, et al. Influence of immersion on respiratory requirements during 30-min cycling exercise. Eur Respir J 1999; 13: 860-866.

5 Krishnan BS, Zintel T, McParland C, Gallagher CG. Evolution of inspiratory and expiratory muscle pressures during endurance exercise. J Appl Physiol 2000; 88: 234-245.

6 Wolf JP, Bréchat PH, Simon-Rigaud ML, Nguyen NU, Regnard J, Berthelay S. Hemodynamic responses to 30-min cycling exercise at 70\% VO2 max both in ambient air and during chest immersion. J Gravit Physiol 1994; 1: 102-103.

7 Mourot L, Bouhaddi M, Gandelin E, et al. Conditions of autonomic reciprocal interplay versus autonomic co-activation: effects on non-linear heart rate dynamics. Auton Neurosci 2007; 137: 27-36.

8 Boussuges A, Molenat F, Grandfond A, et al. Cardiovascular changes induced by cold water immersion during hyperbaric hyperoxic exposure. Clin Physiol Funct Imaging 2007; 27: 268-274.

9 Coulange M. Neuromuscular and cardiovascular consequences of immersion. PhD Thesis. University of AixMarseille 2, France, 2007. 